\title{
Inflammatory Bowel Disease and Sleep Disturbance: As Usual, Quality Matters
}

\author{
Brigida Barberio $^{1} \cdot$ Fabiana Zingone $^{1} \cdot$ Edoardo Vincenzo Savarino $^{1}$
}

Published online: 22 April 2020

(c) Springer Science+Business Media, LLC, part of Springer Nature 2020

Inflammatory bowel disease (IBD), including ulcerative colitis (UC) and Crohn's disease (CD), is associated with a marked reduction of health-related quality of life, severe fatigue, and work impairment, as well as with depression, anxiety, and somatization [1]. Although several studies reported about sleep disturbance in IBD patients and its interaction with disease activity, results of such studies differed to the point of being, in some cases, discordant. A recent study by Marinelli et al. [2] evaluated sleep disturbance in 166 IBD patients, observing that two-thirds of patients with IBD suffer from sleep disturbance. In particular, poor sleep quality is associated with low quality of life, greater disability, and the presence of extraintestinal manifestations, whereas it does not appear to be associated with disease variables such as subtype, disease activity, or type of therapy taken. Conversely, Iskadar et al. [3] found that sleep disturbance was more frequently reported by $\mathrm{CD}$ patients $(n=61)$ than in controls $(n=60)$ and in patients with active $\mathrm{CD}$ compared to those in remission. Likewise, Sobolewska et al. [4] observed that poor sleep can be found in $78 \%$ of clinically active patients $(n=40 / 51)$ in contrast to $35 \%$ of patients in remission $(n=5 / 14)$. On the contrary, Bar-Gil Shitrit et al. [5] demonstrated that poor sleep quality and poor quality of life are present also in patients in clinical remission. Finally, Lee et al. [6] assessed sleep disturbance in IBD patients receiving immunosuppressant and biologic drugs, finding that none of these therapies was predictive of disturbed sleep. Overall, these studies' lack of objective measurements of sleep characteristics including relevant information about sleep efficiency diminished their contributions to this area.

\footnotetext{
Edoardo Vincenzo Savarino

edoardo.savarino@unipd.it

1 Division of Gastroenterology, Department of Surgery, Oncology and Gastroenterology - DiSCOG, University of Padua, Via Giustiniani, 2, 35128 Padua, Italy
}

Sleep disturbance can stimulate immune cell margination and induce inflammatory mediator production. Furthermore, pro-inflammatory cytokines like interleukin-1 (IL-1), tumor necrosis factor- $\alpha$ (TNF- $\alpha$ ), and interferon levels are all increased in the setting of sleep deprivation. Indeed, the circadian clock regulates the activity of the immune system. The central circadian clock is located in the suprachiasmatic nuclei of the anterior hypothalamus, although similar clocks are present in all tissues, including the digestive tract and white blood cells [7]. Circadian gene product expression (mRNA) is reduced in both intestinal biopsies and in peripheral blood mononuclear cells of medically treated IBD patients [8]. In a recent study of Weintraub et al. [9], young, newly diagnosed and untreated patients with IBD have reduced product expression (mRNA) of clock genes in inflamed and non-inflamed intestinal mucosal samples, and also in blood cells, compared with healthy individuals. With this premise, alterations in expression of clock genes might be an early event in IBD pathogenesis. For instance, Liu $\mathrm{X}$ et al. showed in vitro that circadian disruption can downregulate tight junction (TJ) gene expression and thus alter intestinal permeability and exacerbate an immune response being pathophysiological base of IBD beginning or IBD flare [8]. However, to note, the correlation between TJ protein expression and paracellular permeability is not well established in vivo and the mechanistic contribution of increased intestinal paracellular permeability to inflammatory disease and immune function is not well understood.

The twenty-four-hour sleep-wake pattern known as the rest-activity rhythm (RAR) is associated with many aspects of health and well-being. In particular, RARs reflect the effects of both endogenous circadian rhythms and exogenous influences such as light exposure and physical activity; as such, it is considered as an objective measure of sleep efficiency. In this issue of Digestive Disease and Sciences, Conley et al. [10] present their study aimed at describing the sleep characteristics and RAR among 37 adults with IBD (19 UC and 18 CD; 23 IBD in remission and 14 with active 
disease) in order to correlate them to gastrointestinal symptoms and IBD severity. Sleep characteristics and RAR were measured with continuously worn wrist actigraphs in 30-s epochs over 10 days, whereas sleep quality was assessed every morning via an e-mail diary. Moreover, gastrointestinal (GI) symptoms were measured with a baseline survey using GI-PROMIS scales. They found that people with IBD have disrupted actigraph-measured RARs and short and fragmented sleep. Total sleep duration was short, sleep efficiency was low, and wake after sleep onset (WASO) was high in people with active disease and those in remission. Moreover, they found that less robust RARs were associated with more severe GI symptoms, in particular gas/bloating and heartburn/reflux. The authors concluded that people with IBD may have poor objectively measured sleep characteristics and disrupted RARs that are associated with GI symptoms, which in turn may benefit from interventions that promote sleep and regulate their circadian rhythm, in order to improve their quality of life.

The strengths of this study are represented by the objective measurement of sleep characteristics and RARs, using wrist actigraphy over 10 days (including both weekdays and a weekend), and the homogeneity of the study population in terms of race and ethnicity, as the same authors highlighted. These are important points since most of the currently available data, as stated above, are derived from studies where sleep disturbance has not been objectively assessed. Nevertheless, some relevant limitations need to be highlighted. The small sample size with the further stratification of patients according to disease activity using the Physician Global Assessment (PGA) did not allow correct stratification of IBD subjects according to the type of disease (CD vs UC) and IBD-related disease status. Indeed, disease-specific questionnaires for clinical activity (e.g., Harvey-Bradshaw index and partial Mayo score) and objective measures of endoscopic activity (e.g., fecal calprotectin and endoscopy) were not adopted. As a consequence, the authors may not have observed significant differences between those with and without active disease. In turn, the authors were able to find differences only related to general gastrointestinal (e.g., gastroesophageal reflux disease and irritable bowel syndrome) symptoms, since these are not strictly related to inflammation or IBD activity and reflect the global assessment as measured by the PGA. Moreover, the observation that sleep characteristics and RAR may be disrupted in adults with IBD, regardless of disease activity status, cannot be done and suggests the adoption of more specific instruments in order to study the relationship between sleep disturbance and IBD activity. Also, the decision to exclude patients over 60 can be regarded as a limitation given that a large part of IBD population, in particular UC, falls within this age group. Finally, the lack of a control group of healthy subjects or patients with different clinical conditions as well as the lack of an interventional group to support the findings limits the interpretation and generalizability of the results.

In conclusion, further research is needed that measures both objective sleep characteristics and objective disease activity in IBD patients. Only with the results of such studies would it be possible to understand whether people with IBD may benefit from interventions to promote sleep and entrain their circadian rhythm.

Authors' contribution BB, FZ, and ES were involved in study concept, critical review of manuscript, drafting the manuscript, and finalization of manuscript.

Funding None.

\section{Compliance with Ethical Standards}

Conflict of interest Nothing to declare.

\section{References}

1. Marinelli C, Savarino E, Inferrera M, et al. Factors influencing disability and quality of life during treatment: a cross-sectional study on IBD patients. Gastroenterol Res Pract. 2019;2019:5354320.

2. Marinelli C, Savarino EV, Marsilio I, et al. Sleep disturbance in Inflammatory Bowel Disease: prevalence and risk factors-a cross-sectional study. Sci Rep. 2020;10:1-8.

3. Iskandar HN, Linan EE, Patel A. Self-reported sleep disturbance in Crohn's disease is not confirmed by objective sleep measures. Sci Rep. 2020;10:1-9.

4. Sobolewska-Wlodarczyk A, Wlodarczyk M, Banasik J, et al. Sleep disturbance and disease activity in adult patients with inflammatory bowel diseases. J Physiol Pharmacol. 2018;69:423-428.

5. Bar-Gil Shitrit A, Chen-Shuali C, Adar T, et al. Sleep disturbances can be prospectively observed in patients with an inactive inflammatory Bowel Disease. Dig Dis Sci. 2018;63:2992-2997. https:// doi.org/10.1007/s10620-018-5207-0.

6. Lee AJ, Kraemer DF, Kanar O, et al. Immunomodulator and biologic agent effects on sleep quality in patients with inflammatory Bowel disease. Ochsner J. 2018;18:76-80.

7. Cermakian N, Boivin DB. The regulation of central and peripheral circadian clocks in humans. Obes Rev. 2009;10:25-36.

8. Liu X, Yu R, Zhu L, et al. Bidirectional regulation of circadian disturbance and inflammation in inflammatory bowel disease. Inflam Bowel Dis. 2017;23:1741-1751.

9. Weintraub Y, Cohen S, Chapnik N, et al. Clock gene disruption is an initial manifestation of inflammatory Bowel Diseases. Clin Gastroenterol Hepatol. 2020;18:115-122.

10. Conley S, Jeon S, Lehner V, et al. Sleep characteristics and restactivity rhythms are associated with gastrointestinal symptoms among adults with inflammatory Bowel Disease. Dig Dis Sci. (Epub ahead of print). https://doi.org/10.1007/s10620-020-06213 $-6$.

Publisher's Note Springer Nature remains neutral with regard to jurisdictional claims in published maps and institutional affiliations. 